\title{
THE EFFECT OF MAGNETOPHORESIS AND BROWNIAN DIFFUSION ON THE LEVITATION OF BODIES IN A MAGNETIC FLUID
}

\author{
V.G.Bashtovoi ${ }^{1}$, V.K.Polevikov ${ }^{2}$, A.E.Suprun ${ }^{3}$, \\ A.V.Stroots ${ }^{3}$, S.A.Beresnev ${ }^{2}$ \\ ${ }^{1}$ Belarusian National Technical University, \\ 65 Nezavisimosti Ave., Minsk 220013, Belarus \\ ${ }^{2}$ Belarusian State University, 4 Nezavisimosti Ave., Minsk 220030, Belarus \\ ${ }^{3}$ Ferrolabs Inc., 21525 Ridgetop Circle, Suite 260, Dulles VA 20166, USA
}

New aspects related to the redistribution of magnetic particles concentration in a magnetic fluid caused by magnetophoresis and Brownian diffusion in a nonuniform magnetic field are considered. These aspects deal with the influence of these processes on the pressure redistribution and levitation of bodies in a magnetic fluid. It is shown that due to these processes the pressure force acting on bodies changes significantly with time and can be reduced dozens of percent if compared to a homogenous fluid.

Introduction. Since the magnetic fluid is a colloid of magnetic particles, the processes of magnetophoresis and Brownian diffusion have to take place in them in the presence of a strong nonuniform magnetic field [1]. Of special interest is the influence of these processes on the statics of magnetic fluids $[2,3]$. It might be important in many magnetic fluid devices such as sensors [4] and dampers based on the levitation of magnets and nonmagnetic bodies in a magnetic fluid. This effect is caused by the redistribution of the particle concentration $C$ in a magnetic fluid under the action of nonuniform magnetic field. Due to magnetophoresis, magnetic particles are concentrated in those regions, where the magnetic field intensity $H$ is larger. Accordingly, the fluid magnetization $M$ increases in these regions. Since the volume magnetic force acting in the magnetic fluid is defined as a product of fluid magnetization and field gradient, it is determined by these processes and changes with time. Also, the pressure distribution and the pressure force acting on the bodies placed in a magnetic fluid have to change with time and differ from a homogeneous fluid.

1. Governing equations and steady one-dimensional solution. The classical theory of Brownian diffusion [5] is used further. It leads to the following dimensionless equations for the concentration $C[2]$ :

$$
\begin{aligned}
& \frac{\partial C}{\partial t}=\nabla^{2} C-U\left[\Lambda\left(\nabla C \cdot \nabla H+C \nabla^{2} H\right)+C \nabla \Lambda \cdot \nabla H\right] \\
& \Lambda=\operatorname{coth}(U H)-1 /(U H), \quad \mathbf{i}=-\nabla C+U C \Lambda \nabla H .
\end{aligned}
$$

The dimensionless magnetic parameter $U=\mu_{0} m_{\mathrm{m}} H_{0} / k T$ represents the ratio of the potential energy of a particle with magnetic moment $m_{\mathrm{m}}$ in a magnetic field $H_{0}$ to its thermal energy $k T$. Here, $\mu_{0}$ is the magnetic permeability of vacuum, $\Lambda=\Lambda(U H)$ is the Langevin function, and $\mathbf{i}$ is the mass flux density of particles. 
The zero normal component of the mass flux density of particles is taken as the boundary condition and the initial distribution of magnetic particle concentration is assumed constant $(C=1)$.

As it is shown in [2], the stationary mechanical equilibrium in a magnetic fluid exists if the boundaries of the magnetic fluid volume are immovable, isothermal and impermeable for magnetic particles.

One can trace the main features of magnetophoresis and Brownian diffusion effect on the pressure distribution and buoyancy force in a magnetic fluid most distinctly by the example of one-dimensional situation. Let us assume that the parallel-sided layer of a magnetic fluid has a thickness $h$ and is in a nonuniform magnetic field with the gradient perpendicular to the layer. If the $y$-axis is perpendicular to the layer, it means that the boundaries of the layer are $y=0$ and $y=h$, and $H=H(y), C=C(y)$. Let us assume that the boundary $y=0$ is free and the pressure on it is equal to the pressure $p_{0}$ of gas over it, and the boundary $y=h$ is solid. Both boundaries are impermeable for magnetic particles. Let also the magnetic fluid be in the saturation state $\left(M=M_{\mathrm{s}}, \Lambda=1\right)$ and the magnetic field gradient be constant, i.e., $\mathrm{d} H / \mathrm{d} y=G=$ const. Take $h$ as a scale for coordinates, $H_{0}=G h$ as a scale for magnetic field intensity, and $\mu_{0} M_{\mathrm{s}} G h$ as a scale for pressure. The dimensionless governing equations and boundary conditions for this problem with the neglecting of the magnetic pressure drop are

$$
\begin{aligned}
& \frac{\mathrm{d} i_{y}}{\mathrm{~d} y}=\frac{\mathrm{d}}{\mathrm{d} y}\left(\frac{\mathrm{d} C}{\mathrm{~d} y}-U C\right)=0, \quad \frac{\mathrm{d} p}{\mathrm{~d} y}=C \\
& i_{y}=\partial C / \partial y-U C=0 \quad \text { at } y=0 \text { and } y=1 \\
& p=p_{0} \quad \text { at } y=0 ; \quad \int_{0}^{1} C(y) \mathrm{d} y=1 .
\end{aligned}
$$

Corresponding solutions of these equations are

$$
C=U \frac{\exp (U y)}{\exp U-1}, \quad \triangle p(C)=p-p_{0}=\frac{\exp (U y)-1}{\exp U-1} .
$$

It follows that the particle concentration and the pressure exponentially increase in the direction of the magnetic field gradient. In the case of a constant concentration of magnetic particles $(C=1)$ the pressure distribution would be $\triangle p(1)=p-p_{0}=y$. The ratio

$$
F=\frac{\triangle p(C)}{\triangle p(1)}=\frac{\exp (U y)-1}{y(\exp U-1)}
$$

describes the relative change of the pressure distribution and buoyancy force, acting on the nonmagnetic body partly deep into the magnetic fluid, caused by the processes of magnetophoresis and Brownian diffusion. Graphs of the relative buoyancy force are presented in Fig. 1.

First of all it should be noted that these processes lead to the decrease of the pressure and buoyancy force in the magnetic fluid. The decrease is faster when the magnetic parameter $U$ is larger. For instance, $F=0.76$ at $U=1, F=0.54$ at $U=2$, and $F=0.36$ at $U=3$ in the middle part of the layer (at $y=0.5$ ). The lowest $C_{\min }$ and the largest $C_{\max }$ values of the particle concentration take place on the boundaries of the layer $y=0$ and $y=1$, respectively. They are $C_{\min }=0.6$ and $C_{\max }=1.6$ at $U=1, C_{\min }=0.33$ and $C_{\max }=2$ at $U=2, C_{\min }=0.17$ and 


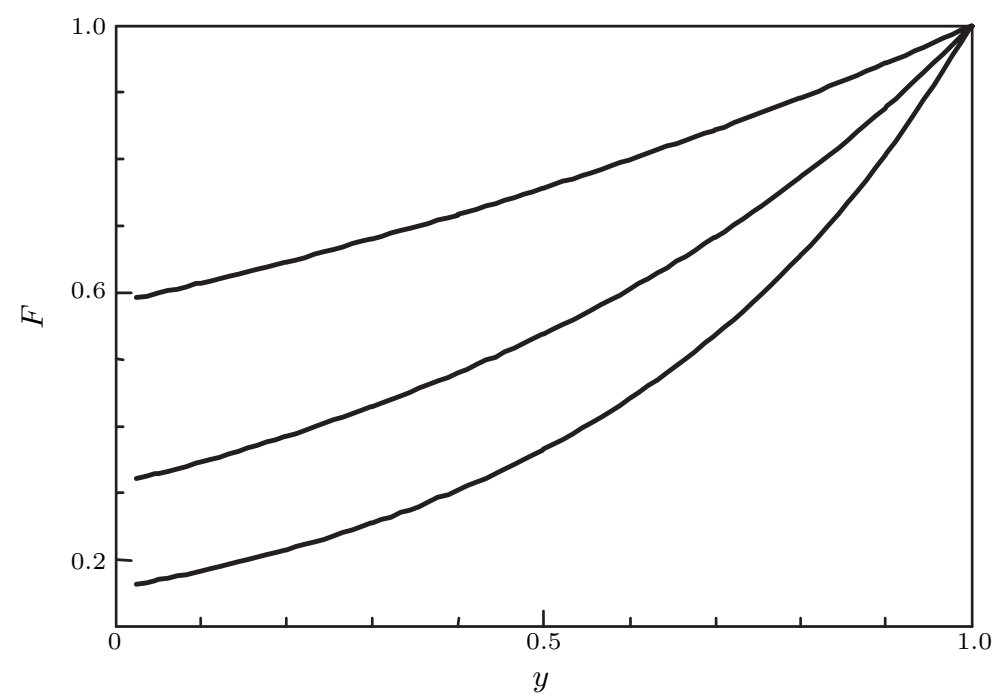

Fig. 1. Dependence of the relative pressure (buoyancy force) on the coordinate for different values of the magnetic parameter: $U=1,2$, and 3 (curves from top down).

$C_{\max }=3$ at $U=3$. At sufficiently high values of the magnetic parameter $U$ the largest value of the particle concentration equals to it, i.e., $C_{\max }=U$.

For any given configuration of the magnetic field $H=H(y)$ and with the Langevin law of magnetic fluid magnetization, the solution of the considered problem is

$$
C=\frac{\sinh (U H)}{H}\left(\int_{0}^{1} \frac{\sinh (U H)}{H} \mathrm{~d} y\right)^{-1}, \quad \triangle p=\frac{C(y)-C(0)}{U}, \quad F=\frac{\triangle p}{y} .
$$

2. Time-dependent two-dimensional problem. Consider the levitation of a nonmagnetic body partly submerged into a magnetic fluid in a nonuniform magnetic field taking into account the above-mentioned processes of magnetophoresis and Brownian diffusion in the two-dimensional geometry. Let us assume a rectangular nonmagnetic body being placed in a rectangular vessel filled with a magnetic fluid, at a distance $h$ from the vessel's bottom as shown in Fig. 2 . The length of the body's side parallel to the vessel's bottom is $L_{b}$. The surface $y=a$ is the free surface of magnetic fluid and the pressure of gas above it is $p_{0}$. The height of the of body's submerged part is $a-h$.

A magnetic field is induced by two opposite poles N, S displaced under the bottom of the vessel at a distance $d$ from it. For instance, they may be the poles of a permanent C-magnet. The pole sizes are $L_{1}$ and $L_{3}$. The magnetic flux through each of them per unit length in the transverse direction is

$$
\Phi_{0}=\int_{L_{1}} H(x,-d) \mathrm{d} x=\int_{L_{3}} H(x,-d) \mathrm{d} x .
$$

Choose $H_{0}=\Phi_{0} / h$ as the scale of the magnetic field intensity. The problem is considered when the magnetic field perturbations caused by the presence of a magnetic fluid are neglected. For the field line function $\Psi(x, y)\left(H_{x}=\partial \Psi / \partial y\right.$, $\left.H_{y}=-\partial \Psi / \partial x\right)$, the Laplace equation

$$
\nabla^{2} \Psi=\frac{\partial^{2} \Psi}{\partial y^{2}}+\frac{\partial^{2} \Psi}{\partial x^{2}}=0
$$




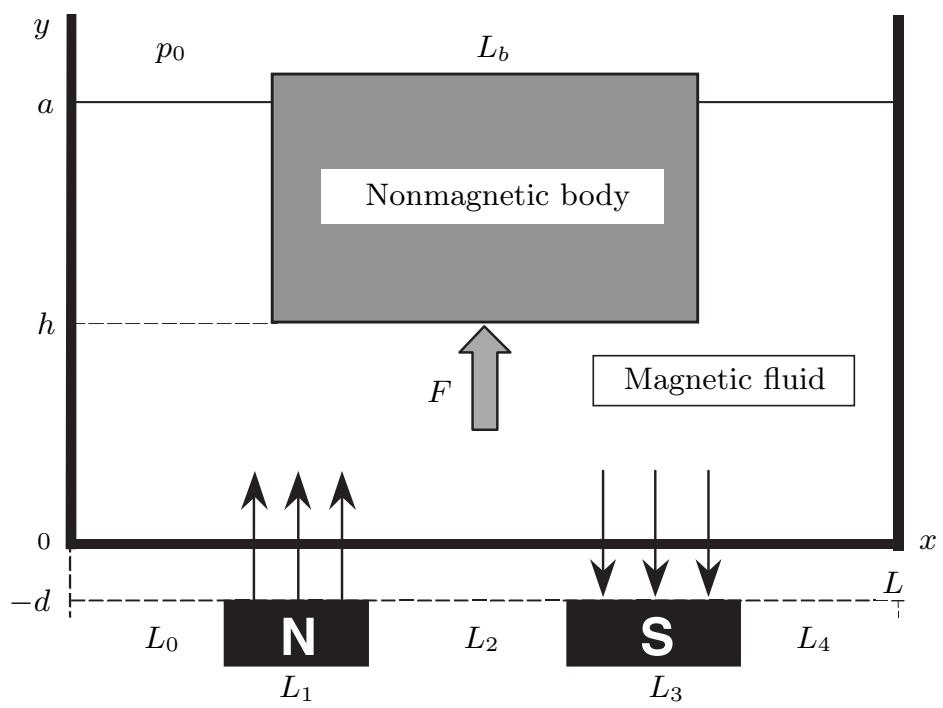

Fig. 2. Geometry of the two-dimensional problem.

is solved with the following boundary conditions at $y=-d$ :

$$
\begin{aligned}
& \partial \Psi / \partial x=0 \Longrightarrow \Psi=\text { const }=0 \quad \text { over the sections } L_{0} \text { and } L_{4} \text {; } \\
& \partial \Psi / \partial y=0, \quad \int(\partial \Psi / \partial x) \mathrm{d} x=\Phi_{0} \quad \text { over the section } L_{1} ; \\
& \partial \Psi / \partial x=0 \Longrightarrow \Psi=\text { const }=\Phi_{0} \quad \text { over the section } L_{2} ; \\
& \partial \Psi / \partial y=0, \quad \int(\partial \Psi / \partial x) \mathrm{d} x=-\Phi_{0} \quad \text { over the section } L_{3} ; \\
& \Psi=0 \quad \text { at } x, y \rightarrow \infty \text {. }
\end{aligned}
$$

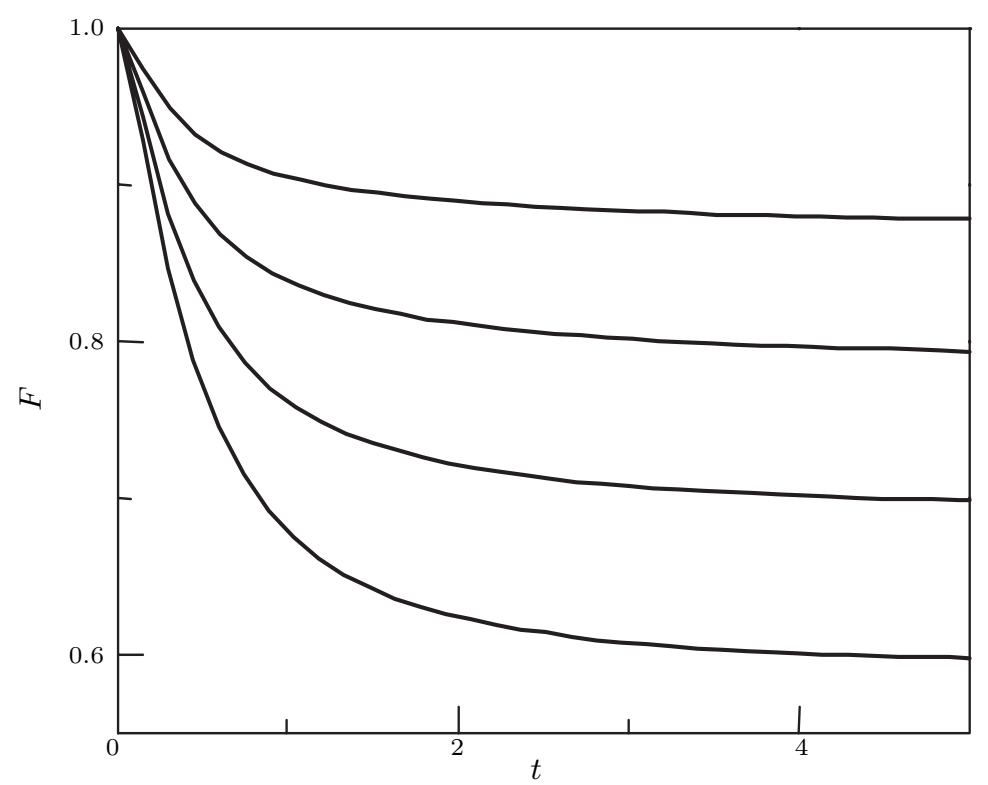

Fig. 3. Time dependence of the relative magnetic buoyancy force resulting from Brownian diffusion: $U=3,4,5$, and 6 (top-down). 


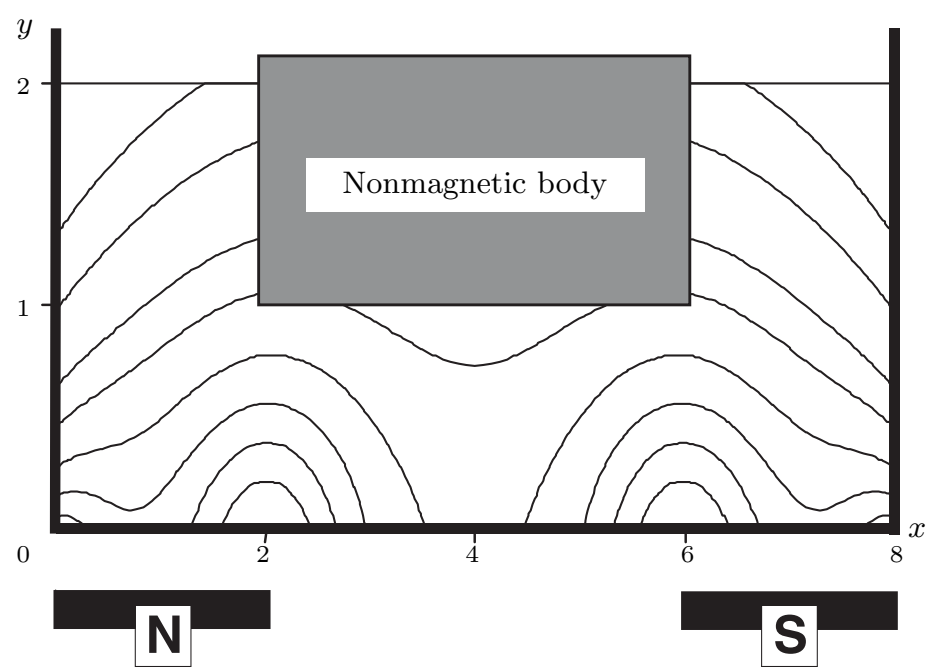

Fig. 4. Isolines of the steady particle concentration in a magnetic fluid at $U=6$. Curves correspond to $C=0.48,0.51,0.63,0.76,1,1.38,2$ and 3.5 (top-down).

A relative magnetic buoyancy force $F$ acting on the nonmagnetic body in a magnetic fluid with magnetization $M=M(C, H)$ is defined as in the previous section and calculated as

$$
F(C)=\frac{1}{F(1)} \int\left(p-p_{0}-0.5 \mu_{0} M_{n}^{2}\right) \mathrm{d} x,
$$

$M_{n}=(M / H) H_{n}$ at the body's bottom at $y=h$. The pressure $p$ is calculated from the statics equation $\nabla p=\mu_{0} M \nabla H, M=M_{\mathrm{s}} C \Lambda$ with the boundary condition $p=p_{0}-0.5 \mu_{0} M_{n}^{2}$ on the free surface $y=a$.

All governing equations are solved numerically by the finite-difference method.

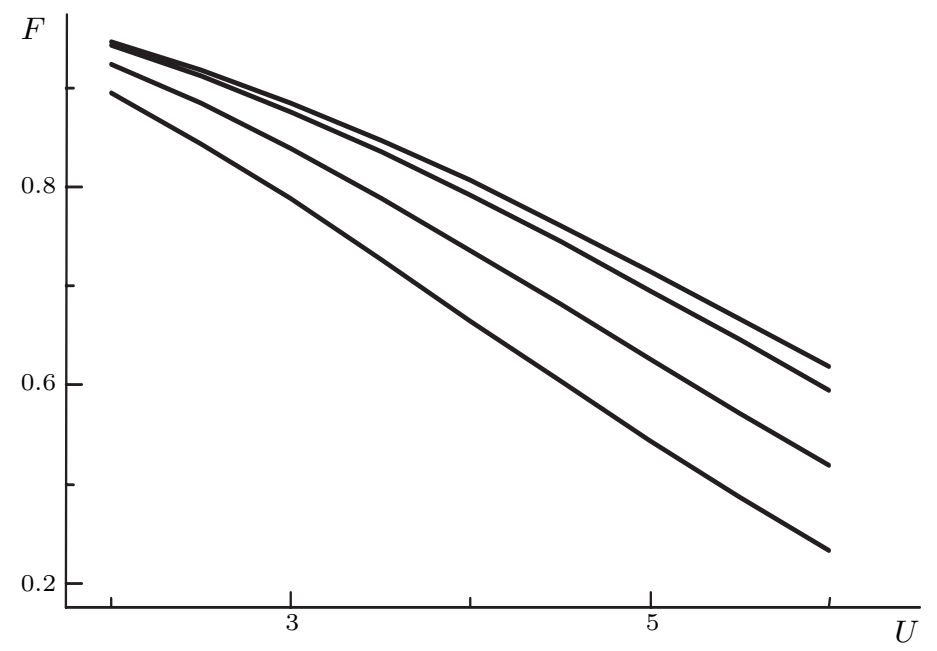

Fig. 5. Dependence of the steady relative magnetic buoyancy force on the magnetic parameter $U$ for different lengths of the nonmagnetic body: $L_{b}=2,4,6$ and 7.92 (topdown). 
The characteristic dimension for the problem is the distance $h$ between the bottoms of the vessel and body, and the characteristic time is $h^{2} / D$. The results given below correspond to the following values of the dimensionless geometrical parameters of the problem: $d=0.27, L_{0}=L_{4}=0, L_{1}=L_{3}=2, L_{2}=4, L=8$, $a=2, L_{b}=4$.

As one can see from the results presented in Figs 3 and 4, the processes of magnetophoresis and Brownian diffusion in a magnetic fluid lead to the decrease of the particle concentration around the body and time decrease of the buoyancy force. It is seen that in this situation the buoyancy force can decrease with time by more than $40 \%$ at $U=6$. This decrease occurs the faster, the higher are the gradients of the magnetic field intensity, i.e., the magnetic parameter $U$ becomes higher.

Fig. 5 demonstrates the significant decrease of the steady relative magnetic buoyancy force with the increase of the magnetic parameter $U$. The decrease is more considerable when the size of the magnetic body $L_{b}$ is larger. Thus, with this size approaching the vessel size $\left(L_{b}=7.92\right)$ and $U=6$, the magnetic buoyancy force decreases more than twice.

It is obvious that the foregoing processes of magnetophoresis and Brownian diffusion in magnetic fluids have an effect on the fluid statics when the magnetic parameter $U$ is larger than unity.

At a typical for the magnetic fluid magnetic moment of particles $m_{\mathrm{m}}=2.5$. $10^{-19} \mathrm{~J} / \mathrm{T}$ and at room temperature $T=300 \mathrm{~K}$, the quantity $U=1$ is valid at a magnetic field intensity of about $10 \mathrm{kA} / \mathrm{m}$. In real magnetic fluid devices such as seals, dampers and sensors using strong permanent magnets the intensity of magnetic field can be much higher (up to $100 \mathrm{kA} / \mathrm{m}$ and more) and $U$ can attain several unities and even dozens.

In conclusion it may be noted that, as it is shown in [3], the theory presented here describes well the processes in magnetic fluid seals and the results predicted above were observed distinctly by the authors experimentally in a magnetic fluid damper.

Acknowledgements. The authors thank the Belarusian Republic Foundation for Fundamental Researches for support.

\section{REFERENCES}

[1] R.E. Rosensweig. Ferrohydrodynamics (Cambridge University Press, New York, 1985).

[2] V.G. Bashtovoi, V.K. Polevikov, A.E.Suprun., A.V.Stroots, S.A. BerESnev. Influence of Brownian diffusion on statics of magnetic fluid. Magnetohydrodynamics, vol. 43 (2007), no. 1, pp. 17-25.

[3] V.G. Bashtovoi, V.K. Polevikov, A.M. Algadal. The effect of diffusion processes on the statics of magnetic fluids. Proceedings of the National Academy of Sciences of Belarus, (2006), no. 3, pp. 42-48 (in Russ).

[4] A.E. Suprun, D.V. Simonenko, Yu.I. Romanov. Computer input device. US Patent, 6, 731, 268 (2004), no. 2, pp. 1150-1159.

[5] L.D. Landau E.M. Lifshitz. Fluid Mechanics. (Pergamon Press, London, 1959).

Received 02.04.2008 